\title{
Estudio piloto sobre políticas de salud bucal y VIH
}

\author{
Irene Tamí-Maury, ${ }^{1}$ Miguel Agüero ${ }^{2}$ y Saskia Estupiñán-Day ${ }^{3}$
}

Forma de citar Tamí-Maury I, Agüero M, Estupiñán-Day S. Estudio piloto sobre políticas de salud bucal y VIH. Rev Panam Salud Publica. 2009;26(6):536-40.

RESUMEN Objetivo. Obtener y evaluar información sobre las politicas nacionales para la atención odontológica de los pacientes con VIH/Sida en los países de las Américas.

Métodos. Se llevó a cabo un estudio transversal y descriptivo mediante un cuestionario en línea diseñado para ser completado por los encargados nacionales de la salud bucal en la Región.

Resultados. De los 38 países invitados a participar, solamente $12(31,6 \%)$ completaron el cuestionario. De esos 12 países, ocho $(66,7 \%)$ informaron tener politicas nacionales y estrategias para proveer salud bucal al paciente con VIH/Sida. Las lineas de investigación más desarrolladas por los países durante los últimos cinco años fueron i) prácticas de control de infecciones, ii) prevalencia de lesiones bucales asociadas a VIH/Sida, iii) discriminación del paciente seropositivo en la consulta odontológica y iv) cobertura odontológica para personas infectadas. Entre las necesidades informadas por los encargados nacionales del sector, se encuentran el incrementar el conocimiento sobre las buenas prácticas de control de infecciones y los avances en materia VIH, la creación de espacios para intercambio de ideas y de foros de discusión en línea, y contar con información sobre fuentes de financiamiento para estudios y proyectos.

Conclusiones. A pesar del incremento en la incidencia y prevalencia del VIH/Sida en las Américas, en general no parece haber políticas, estrategias ni programas que aborden idóneamente el problema de la salud bucal y la infección por este virus. Es imperante establecer una comunicación efectiva entre investigadores y hacedores de políticas dentales sanitarias, a fin de confrontar una epidemia que requiere cada vez más de profesionales odontólogos altamente capacitados para ofrecer una mejor calidad de vida a la población afectada.

Palabras clave Legislación en salud; odontología; políticas de salud; salud bucal; sida; VIH; Américas.

La epidemia del virus de inmunodeficiencia humana (VIH) se ha convertido en un desastre social que afecta particularmente a los países en desarrollo de América Latina y el Caribe, África y el sureste asiático. El último informe del Pro-

1 Universidad de Alabama, Birmingham, Estados Unidos. La correspondencia debe dirigirse a Irene Tamí-Maury, University of Alabama at Birmingham, NB 1014, 1530 3rd Avenue South, Birmingham, AL 35294-1210. USA. Correo electrónico: irene.tami@fulbrightmail.org

2 Voluntario de la Organización Panamericana de la Salud (OPS), Estados Unidos.

3 Programas Especializados y Salud de las Poblaciones Vulnerables, OPS, Estados Unidos. grama Conjunto de las Naciones Unidas sobre el VIH/Sida (ONUSIDA) estimó que hacia fines de 2007 unas 33,2 millones de personas estaban infectadas con este virus. En las Américas esa cifra alcanzaba a 2,6 millones de personas, de las cuales 1,6 millones correspondían a América Latina, 1,3 millones a América del Norte y cerca de 230000 al Caribe $(1,2)$. En este continente, cada año alrededor de 163000 personas contraen infección por VIH, mientras que otras 90000 fallecen a causa de Sida, en la mayoría de los casos debido a falta de acceso a servicios de prevención y tratamiento adecuados (1).
Dentro de esta carencia de servicios destaca el problema de los pacientes seropositivos al virus que requieren de atención odontológica. Es lógico pensar entonces que tanto los servicios públicos de salud bucal como los profesionales odontológicos pueden contribuir significativamente al control de la epidemia de VIH/Sida a través de programas que incluyan educación sobre promoción sanitaria, cuidados del paciente, control efectivo de la infección y vigilancia epidemiológica.

En 2003 la Organización Mundial de la Salud (OMS) publicó el Informe sobre la 
salud bucodental en el mundo, donde se enfatiza la importancia de promocionar la salud bucal, considerada como una parte integral de la salud general y un componente esencial para una buena calidad de vida. Se ha observado, por ejemplo, que entre 40 y $50 \%$ de personas VIHpositivas desarrolla algún tipo de lesión bacteriana, micótica o viral en la boca durante el curso de la infección por VIH. De hecho, las manifestaciones bucales asociadas al VIH/Sida son consideradas un desafío importante para mejorar la salud en el futuro, particularmente en los países en desarrollo (3-5). Sin embargo, en América Latina y el Caribe los esfuerzos tanto de los gobiernos nacionales como de los organismos internacionales y organizaciones de la sociedad civil han sido débiles y dispersos en sus objetivos y cobertura con respecto a la salud bucal y dental de los pacientes VIH/Sida.

En 1995 la Organización Panamericana de la Salud (OPS) publicó La salud bucodental: repercusión del VIH/Sida en la práctica odontológica, dirigida principalmente a los profesionales de servicios dentales, donde se incluyen valiosas recomendaciones para complementar los modelos propuestos por el sector profesional y gubernamental de cada país (6). No obstante, hasta hoy en muchos países tales modelos de atención o no existen o siguen siendo insuficientes para encarar las consecuencias del VIH/Sida en la salud odontológica de la Región.

Con algunas excepciones (7-9), en general en los países de la Región no existen políticas nacionales para la atención odontológica del paciente con VIH/ Sida ${ }^{4}$ y donde existen, no son efectivas porque carecen de programas definidos y estrategias coherentes con la realidad de cada país ${ }^{5}$ (10). Incluso en países donde la investigación sobre la epidemia de VIH/Sida ha sido pionera a nivel mundial, como es el caso de Estados Unidos, muchos de los pacientes VIHpositivos no reciben los cuidados odontológicos necesarios (11).

Con bastante frecuencia, las iniciativas para proveer atención odontológica al paciente con VIH en la Región provienen de instituciones académicas, gremiales $u$

Organización Panamericana de la Salud. La salud oral para el caribeño. La Habana, abril de 2004. (Documento fotocopiado).

5 Perú. Programas de gobierno en población 20062011. Lima, enero-junio de 2006. (Documento fotocopiado). organizaciones no gubernamentales, pero estos esfuerzos, sin duda loables, también son intermitentes y llevados a cabo sin una clara coordinación con los programas y estrategias nacionales $(10,12)$. Esta falta de articulación en los esfuerzos, aunada a los problemas de discriminación, inequidad socioeconómica y escasa disponibilidad de servicios que afectan a algunos países, se traducen solamente en serias dificultades que debe enfrentar el paciente VIH positivo para acceder a la consulta odontológica (13-17).

También se observa un divorcio entre la comunidad científica y los hacedores de políticas sanitarias (18). Aun cuando se han publicado numerosos trabajos científicos relacionados con la salud bucal y la infección por VIH/Sida en la Región, por lo general los hallazgos de estas investigaciones no son incorporados a las políticas nacionales y las recomendaciones son escasamente integradas en las decisiones sanitarias. Ya ha sido mencionado por otros autores que la irrelevancia de las investigaciones con respecto a las necesidades sanitarias del país, la incapacidad de los directivos sanitarios en absorber hallazgos científicos importantes, la ausencia de participación comunitaria en la búsqueda de soluciones y el escaso presupuesto sanitario de muchos países de la región muchas veces impiden poner en práctica los hallazgos de investigación en los programas y estrategias sanitarias $(19,20)$.

Con el propósito de obtener mayor información sobre las políticas y programas para la atención odontológica del paciente con VIH/Sida, en julio y agosto de 2007 la División de Salud Bucal de la OPS realizó un estudio piloto con la participación de los encargados de la salud dental en los países de la Región.

\section{MATERIALES Y MÉTODOS}

Se llevó a cabo un estudio transversal y descriptivo, instrumentado mediante la creación de un cuestionario en línea para ser completado por profesionales participantes. El cuestionario, no validado previamente por tratarse de un proyecto piloto, fue redactado en español y en inglés y constó de 10 preguntas tanto abiertas como cerradas. Fue desarrollado mediante el software SurveyMonkey.com $@$, un programa que permite elaborar cuestionarios multilingües de manera rápida y sencilla. Cuenta además con opciones generales para validar textos y exigir un número determinado de respuestas, manteniendo a la vez la seguridad y privacidad de la información proporcionada.

Las preguntas incluidas en el cuestionario se relacionaron con i) sistema de salud bucal, incluidos el número de escuelas/facultades de odontología y de odontólogos en el país; ii) la prevalencia de VIH/Sida; iii) la disponibilidad de políticas, estrategias, manuales y protocolos para el cuidado odontológico de pacientes con VIH/Sida; iv) publicaciones científicas sobre salud bucal y VIH/Sida; v) instituciones y centros del país o región que proveen servicios a personas con VIH/Sida, y vi) necesidades reconocidas por los encuestados acerca de la salud bucal y la infección con VIH/Sida.

El acceso a este cuestionario de enfoque cualitativo fue otorgado mediante invitación por correo electrónico a cada encargado nacional de la salud dental, con un plazo de cuatro semanas para responder todas las preguntas. Como previsión para casos en que los participantes tuvieran dificultades con el llenado del cuestionario, se mantuvo disponible un servicio de apoyo técnico que fue brindado por correo electrónico o vía telefónica. En los casos en que el participante lo prefiriera, el cuestionario fue enviado por facsímile.

Los análisis descriptivos fueron realizados con la ayuda del paquete estadístico SPSS para Windows, versión 11.5 (SPSS Inc., IL, USA). En total 38 países fueron invitados a participar (cuadro 1).

\section{RESULTADOS}

Dado que solamente $12(31,6 \%)$ de los 38 países invitados completaron el cuestionario, no fue posible preparar un censo adecuado del número de escuelas y facultades de odontología, ni del número de dentistas con que cuenta la Región. Las respuestas a la pregunta sobre el "número de personas infectadas con VIH/Sida en su país" fueron comparadas con las estimaciones de ONUSIDA para el año 2006 (21) con el objeto de evaluar su validez. De los 12 países que completaron el cuestionario, solo tres (25\%) notificaron estimaciones confiables sobre prevalencia de VIH/Sida. La razón odontólogos/personas con VIH/ Sida fue calculada para los 12 países que respondieron el cuestionario (cuadro 2), tomando en consideración el número de 
CUADRO 1. Países contactados para participar en un estudio sobre políticas de salud bucal y virus de la inmunodeficiencia humana, por regiones

\begin{tabular}{llll}
\hline \multicolumn{1}{c}{ Caribe } & América Central & América del Sur & América del Norte \\
\hline Antigua y Barbuda & Belice & Argentina & Canadá \\
Bahamas & Costa Rica & Bolivia & Estados Unidos \\
Barbados & El Salvador & Brasil & México \\
Bermuda & Guatemala & Chile & \\
Islas Vírgenes Británicas & Honduras & Colombia & \\
Islas Caimán & Nicaragua & Ecuador & \\
Cuba & Panamá & Guyana & \\
Dominica & & Paraguay & \\
República Dominicana & & Perú & \\
Granada & & Suriname & \\
Jamaica & & Uruguay & \\
Montserrat & & Venezuela & \\
Saint Kitts y Nevis & & \\
Santa Lucía & & \\
San Vicente y las Granadinas & & \\
Trinidad y Tabago & & \\
\hline
\end{tabular}

CUADRO 2. Razón odontólogos/población infectada con VIH/Sida para varios países de las Américas

\begin{tabular}{ll}
\hline \multicolumn{1}{c}{ País $^{a}$} & Razón \\
\hline Suriname & $1: 132$ \\
Guatemala & $1: 26$ \\
Ecuador & $1: 19$ \\
Panamá & $1: 8$ \\
Perú & $1: 5$ \\
Canadá & $1: 3$ \\
Brasil & $1: 3$ \\
Chile & $1: 3$ \\
Islas Caimán & $1: 3$ \\
Uruguay & $1: 2$ \\
Costa Rica & $1: 2$ \\
El Salvador & $1: 2$ \\
\hline
\end{tabular}

Fuente: realizado por los autores a partir del número de odontólogos informado por los encargados nacionales de la salud bucal y la población infectada con $\mathrm{VIH} /$ Sida por país/ región según estimaciones de ONUSIDA, 2006 (21).

a Los países incluidos son aquellos que completaron el cuestionario propuesto.

odontólogos informado en el cuestionario por cada encargado nacional de salud bucal y la población infectada con VIH/ Sida por país/región según las estimaciones de ONUSIDA 2006 (21). Ocho $(66,7 \%)$ de los 12 países que contestaron el cuestionario informaron tener políticas y estrategias para proveer salud bucal a las personas infectadas con VIH/ Sida, aunque sólo siete $(58,3 \%)$ cuentan con manuales y protocolos específicos para tal fin.

En referencia a las líneas de investigación que los participantes señalaron como las más desarrolladas en los últimos cinco años caben destacar, en orden decreciente, prácticas de control de infecciones en el consultorio dental, prevalencia de lesiones bucales en las personas con VIH/ Sida, discriminación de personas con $\mathrm{VIH} /$ Sida en la consulta odontológica y cobertura dental para pacientes con esta infección. Con respecto a las necesidades, las respuestas incluyeron incrementar el conocimiento acerca de las prácticas de control de infecciones y avances en VIH, facilitar espacios para intercambio de ideas entre las autoridades nacionales y los profesionales de la salud dental, establecer foros de discusión en línea, y proveer información sobre fuentes de financiamiento para estudios y proyectos.

\section{DISCUSIÓN}

Debido a que apenas 31,6\% de los países invitados a participar respondieron al cuestionario del estudio piloto, la información obtenida sobre las políticas nacionales para la atención odontológica del paciente con VIH/Sida y la realidad odontológica de la Región frente a la epidemia VIH/Sida fue limitada. No obstante, al evaluar la baja tasa de participación hay que considerar cierto impacto que pudieran haber tenido las barreras del idioma - para los países de habla francesa y portuguesa - y la falta de acceso a Internet (22). Aun así, también es cierto que en la mayoría de los países que no completaron el cuestionario el idioma oficial es el español o el inglés, precisamente los idiomas en que los cuestionarios fueron preparados. En relación con la falta de acceso a Internet, los participantes tenían la opción de usar facsímile y también el correo convencional.
Con todo, sería prematuro pensar que la baja participación es únicamente reflejo de poco interés en el problema por parte de los programas de salud bucal nacionales en crear estrategias acordes con la situación de la epidemia y contribuir con los esfuerzos que promueven los organismos internacionales. Hay que reconocer por ejemplo que los recursos disponibles para el sector salud en la mayoría de los países pudieran ser un factor determinante en nuestros resultados, los cuales por lo demás solamente podrían estar reflejando la realidad sanitaria de la Región, especialmente en frente a la epidemia VIH/Sida.

Sorprende sin embargo que nueve $(75 \%)$ de los encargados nacionales de la salud dental que participaron en este estudio desconozcan la realidad de la epidemia VIH/Sida en su país. Una explicación podría ser que muchos países de la Región no cuentan con sistemas de vigilancia epidemiológica efectivos que permitirían conocer con precisión la magnitud real de epidemia del VIH/ Sida (23-26). Claro que tampoco se puede descartar la posibilidad de que el problema radique en la falta de integración efectiva entre el programa odontológico nacional y el programa de VIH/Sida de cada país/región.

Un hallazgo del estudio es la situación alarmante que experimenta Suriname con respecto a su fuerza laboral odontológica, con una razón de un odontólogo por cada 132 personas infectadas con VIH/Sida, sin contar el resto de la población que requiere también de atención odontológica (lo que representa aproximadamente un odontólogo por cada 11513 habitantes del país). Tal escasez de odontólogos ha sido paliada acertadamente desde hace más de dos décadas mediante cursos para el entrenamiento en técnicas dentales de enfermeros y personal auxiliar de salud, con el apoyo de la asociación de odontólogos nacionales (27).

Estudios realizados en surinameses que padecen ciertas enfermedades crónicas como cáncer, diabetes e hipertensión, han resaltado la necesidad de mejorar la comunicación entre médicos y odontólogos a fin de proveer una atención más efectiva a estos pacientes (28). Tal necesidad se aplica igualmente a los pacientes con VIH/Sida, cuya supervivencia ha sido prolongada gracias a los tratamiento anti-retrovirales. En la estrategia 2005-2010 para la salud bucal en la región de las Américas, publicada por la 
OPS, se asigna particular énfasis al desarrollo de políticas y proyectos locales, nacionales y regionales que contribuyan a reducir el impacto de una condición bucal pobre en la salud general de las personas, incluyendo aquellas que viven con VIH/Sida (29).

Si bien en LILACS y MedLine, las dos bases de datos más importantes de la Región, no se han encontrado muchos informes sobre políticas sanitarias de salud bucal para personas positivas al VIH, no debe descartarse la posibilidad de que haya países que posean estrategias y programas dirigidos de esta índole que lamentablemente no han sido conocidos en la región.

Una limitante significativa de este estudio fue la imposibilidad de validar el instrumento de recolección de datos, dado que no permitió comparar sus resultados con los de otros estudios. También el hecho de que solamente 12 de los 38 países contactados hayan respondido no permite efectuar generalizaciones de los resultados. Aun así, dado que hasta la fecha no parece haber estudios que hayan abordado el problema de los servicios de salud bucal en personas infectadas con VIH/Sida, el presente trabajo podría significar un primer paso que allane el camino para nuevas investigaciones.

En futuras investigaciones sería recomendable producir los contenidos $\mathrm{y}$ otros materiales del estudio en español, francés, inglés y portugués.

Adicionalmente, debido que los programas de salud bucal y los programas de VIH/Sida parecen desligados en algunos países de la Región, se recomienda llevar a cabo estudios de corte cualitativo que contribuyan a que entidades gubernamentales, no gubernamentales y académicas unan esfuerzos para consolidar una estrategia nacional y promover la comunicación entre las áreas de medicina y odontología.

Si bien la información sobre políticas y proyectos para la atención odontológica del paciente con VIH/Sida proporcio- nada por los participantes en el estudio fue limitada, queda claro que hace falta brindar respaldo permanente a los esfuerzos realizados por los países para dar soluciones a este problema de salud pública, incluyendo actividades de monitoreo y medición de progresos. Como estrategia a corto plazo, es importante que los organismos internacionales y regionales competentes identifiquen a los países que hayan demostrado un cambio positivo en el cuidado de la salud bucal en personas infectadas con VIH/Sida. Más aún, estas buenas prácticas deben ser difundidas para estimular y ofrecer oportunidades de aprendizaje a otros países de la Región.

Agradecimiento. Los autores desean expresar su gratitud a Isabel Cristina Garcés, de la Universidad de Alabama, Birmingham, Estados Unidos, por sus valiosos comentarios y sugerencias para este artículo.

\section{REFERENCIAS}

1. The United Nations Joint Programme on HIV/AIDS. AIDS epidemic update: December 2007. Geneva: UNAIDS; 2007.

2. The Henry J. Kaiser Family Foundation. The Global HIV/AIDS Epidemic: June 2008. Hallado en: http://www.kff.org/hivaids/ upload/ 3030-11.pdf. Acceso el 10 de Octubre de 2009.

3. World Health Organization. The World Oral Health Report 2003. Geneva: WHO; 2003.

4. Ferreira S, Noce C, Junior AS, Goncalves L, Torres S, Meeks V, et al. Prevalence of oral manifestations of HIV infection in Rio De Janeiro, Brazil, from 1988 to 2004. AIDS Patient Care and STDS. 2007;21(10):724-31.

5. Petersen P. Policy for prevention of oral manifestations in HIV / AIDS: The approach of the WHO global oral health program. Adv Dent Res. 2006;19(1):17-20.

6. Organización Panamericana de la Salud (OPS). La salud buco-dental: Repercusión del $\mathrm{VIH} /$ Sida en la práctica odontológica. Washington, DC: OPS; 1995.

7. Ministerio de Salud de El Salvador. Alcances y logros de la lucha contra el VIH/Sida en el área odontológica. San Salvador: Ministerio de Salud; 2006.

8. Nunes M, Pereira M, Alves R, Leles C. A proposta da educação permanente em saúde na formação de cirurgiões-dentistas em DST/ HIV/AIDS. Interface Comun Saude Educ. 2008;12(25):413-20.

9. Canadian Public Health Association. HIV and oral health-care and confidentiality. Hallado en: http://www.cpha.ca/en/portals/hiv/ article01.aspx. Acceso el 10 de Octubre de 2009.
10. Acosta de Velásquez B. Atención al paciente VIH/Sida: Legislación y bioseguridad odontológica en Colombia. Acta Bioeth. 2006;12(1): 23-8.

11. Research and Development Corporation. Do people with HIV get the dental care they need? RAND Corporation research brief series. RAND; 2005. Hallado en: http://www. rand.org/pubs/research_briefs/RB9067/ RAND_RB9067.pdf. Acceso el 10 de Octubre de 2009.

12. Garrido E, Tovar V, Guerra ME, Carvajal A, León JR. Programa odontológico educativo en salud bucal dirigido a madres VIH/Sida. Acta Odontológica Venezolana. 2008;46(1). Hallado en: http:/ / www.actaodontologica.com/ ediciones/2008/1/programa_odontologico_ educativo_salud_bucal.asp. Acceso el 10 de Octubre de 2009.

13. Dávila M, Gil M. Nivel de conocimiento y actitud de los odontólogos hacia portadores de VIH/Sida. Acta Odontológica Venezolana. 2007;45(2). Hallado en: http://www.acta odontologica.com/ediciones/2007/2/nivel conocimiento_actitud.as. Acceso el 10 de Octubre de 2009.

14. Rosa M, Coelho Â, Albuquerque S. Atitude dos cirurgiões-dentistas de João Pessoa-PB frente a AIDS. Odontol Clin Cient. 2006;5(2): 123-8.

15. Robinson P. Implications of HIV disease for oral health services. Adv Dent Res. 2006;19(1): 73-9.

16. García-Sánchez C, Sierra-Gallego J, VidalQuintero Y, Contreras-González D, MartínezHerrera E, Agudelo-Suárez A. Percepción de personas VIH/SIDA sobre la atención odon- tológica. Rev Cub Salud Pública. 2008;45(1). Hallado en: http://bvs.sld.cu/revistas/est/ vol45_1_08/est05108.htm. Acceso el 10 de Octubre de 2009.

17. Irigoyen-Camacho $M$, Zepeda-Zepeda $M$, Maupome G, Lopez-Camara V. Attitudes of a group of Mexico City residents toward HIV/AIDS in the dental office. Am J Infect Control. 2003;31(4):231-6.

18. Macías-Chapula C. Hacia un modelo de comunicación en salud pública en América Latina y el Caribe. Rev Panam Salud Publica. 2005;18(6):427-38.

19. Organización Panamericana de la Salud. Mayor respaldo científico para evaluar la eficacia de los programas de salud pública. Rev Panam Salud Publica. 2005;18(6):448-9.

20. Kothari A, Birch S, Charles C. Interaction and research utilization in health policies and programs: Does it work? Health Policy Plan. 2005;71(1):117-25.

21. The United Nations Joint Programme on HIV/AIDS. Report on the global AIDS epidemic: A UNAIDS 10th anniversary special edition. Geneva: UNAIDS; 2006.

22. Jacobs E, Chen AH, Karliner LS, Agger-Gupta $\mathrm{N}$, Mutha $\mathrm{S}$. The need for more research on language barriers in health care: A proposed research agenda. Milbank Q. 2006; 84(1):111-33.

23. Cabrera D, Sánchez $S$, Jave $O$, Carrión $M$, Jamanca R. Infección VIH/Sida en la jurisdicción de la Dirección de Salud de Lima Ciudad, 1984-2004. Rev Peru Med Exp Salud Pública. 2005;22(4):275-80.

24. Organización Panamericana de la Salud. Sida en Latinoamérica, ¿una epidemia controlable? 
[reseña]. Hallado en: http:/ /www.paho.org/ spanish/dd/pin/ps040723.htm. Acceso el 10 de Octubre de 2009.

25. Soto RJ. El VIH/Sida en Honduras, "Características de la Epidemia". Hallado en: http:// rds.hn/index.php?documento=679. Acceso el 10 de Octubre de 2009.

26. García-Abreu A, Moguer I, Cowgill K. El $\mathrm{VIH} /$ Sida en países de América Latina. Los retos futuros. Washington, DC: Organización Panamericana de la Salud; 2004.
27. Guile E, Hagens E, Miranda J. Dental nursing in Suriname: Training and deployment. J Dent Educ. 1981;45(3):156-60.

28. Eyre H, Kahn R, Robertson RM, the ACS/ ADA/AHA Collaborative Writing Committee. Preventing cancer, cardiovascular disease, and diabetes: A common agenda for the American Cancer Society, the American Diabetes Association, and the American Heart Association. Circulation. 2004;291(24): 2990-3.
29. Pan American Health Organization (PAHO). Strategy for improving general health in the Americas through critical advancements in oral health. The way forward: 2005-2015. Washington, DC: PAHO; 2005.

Manuscrito recibido el 7 de noviembre de 2008. Aceptado para publicación, tras revisión, el 24 de mayo de 2009.

ABSTRACT Objective. To obtain and evaluate information on national oral health policies regarding patients with HIV/AIDS in the countries of the Americas.

\section{Pilot study of oral health policy and HIV}

Methods. A cross-sectional descriptive study was conducted using an online questionnaire designed to be completed by the national oral health officials in the Region. Results. Of the 38 countries that were asked to participate, only $12(31.6 \%)$ completed the questionnaire. Of these 12, eight (66.7\%) reported having national policies and strategies in place to promote oral health for the HIV/AIDS patient. Areas of study most explored by the countries over the last 5 years have been: (a) infection control practices; (b) the prevalence of HIV / AIDS-related oral lesions; (c) discrimination toward the HIV-positive patient during dental consultation; and, (d) dental coverage for infected individuals. Among the needs reported by the national officials of this sector were: increasing knowledge of good infection-control practices and HIV advances, development of forums for exchanging ideas and holding online discussions, and having access to information on sources of funding for studies and projects.

Conclusions. Despite increased HIV/AIDS incidence and prevalence in the Americas, in general, there do not seem to be any model policies, strategies, or programs that address the issue of oral health and HIV infection. Establishing effective communication between dental health researchers and policymakers is imperative to confronting this epidemic, which increasingly requires more from the highly-trained dental professionals who offer quality of life to the affected population.

Key words Legislation, health; dentistry; health policy; oral health; acquired immunodeficiency syndrome; HIV; Americas. 